\title{
Distribución Normal: Análisis histórico-epistemológico e implicaciones didácticas
}

\author{
Normal Distribution: \\ Historical-epistemological analysis and didactic implication
}

\section{NEEMIAS LEMUS-CORTEZ}

C. Magíster en Didáctica de la Matemática

Pontificia Universidad Católica de Valparaíso

neemias.lemus.c@mail.pucv.cl

\author{
JAIME HUINCAHUE \\ Dr. en Didáctica de la Matemática \\ Vicerrectoría de Investigación y Postgrado \\ Universidad Católica del Maule \\ jhuincahue@ucm.cl
}

\section{RESUMEN}

Al estudiar los comportamientos en la dispersión de datos, es posible reconocer a la Distribución Normal (DNor) como un objeto matemático/estadístico de relevancia que amerita ser profundizado en su tratamiento escolar. Este artículo propone, desde una dimensión histórico-epistemológica, analizar y evaluar una ruta epistémica para una aproximación conceptual, con el fin de propiciar su aprendizaje desde un enfoque multidisciplinar. Al respecto, se visualiza a la curva de los errores como una aproximación de la DNor, centrando el análisis en el comportamiento del error del dato por sobre el dato, y se obtiene una aproximación conceptual ajena, singular e innovadora a la promocionada curricularmente. Para ello, se decide situar como marco conceptual un enfoque tradicional de la modelación matemática desde la didáctica de la matemática, clarificando su uso en la saturación de oxígeno en la sangre. Utilizando la ingeniería didáctica como diseño metodológico, se analizan las respuestas de la tarea de modelación propuesta y aplicada en estudiantes de 17 a 18 años en la Región del Libertador Bernardo O'Higgins. Los resultados muestran que la tarea de modelación propicia el aprendizaje de la DNor, siendo hoy una propuesta que amplía didácticamente los procesos de enseñanza y aprendizaje de la DNor.

Palabras clave: Distribución Normal, epistemología, historia, modelación matemática, saturómetro. 


\section{ABSTRACT}

Examinating the behaviors in the data dispersion, it is possible to recognize the Normal Distribution as a mathematic/statistic object of relevance to deepen its scholastic processing. This article proposes, from a historical-epistemological approach, to analyze and suggest an epistemic route for a conceptual approximation, with the aim of encouraging and multidisciplinary approach. Related to this, it is recognized the normal probability plot as an approximation to the Normal Distribution, centering the analysis in the behavior of the error's data beyond the data, getting a conceptual approximation external, singular and innovating from the one promoted in terms of curriculum. For that it is suggested to contextualize as a conceptual framework a traditional approach of the mathematic modeling from the mathematic didactic, clearing the use of the oxygen blood's saturation. Using the didactic engineering as qualitative methodological design, the answers to the modeling task applied in students from 17 to 18 years in the Libertador Bernardo O'Higgins region are analyzed. The results show that the modeling task is conductive to the learning of Normal Distribution, being today a proposal that increases didactically not only the teaching, but also the learning of the Normal Distribution.

Key words: Normal Distribution, epistemology, history, mathematical modelling, saturometer.

\section{INTRODUCCIÓN}

Actualmente, los esfuerzos disciplinares de las comunidades especializadas en la enseñanza y el aprendizaje de las matemáticas (llámese didáctica de la matemática, educación matemática o matemática educativa) conciben que una de las aproximaciones para lograr fortalecer y mejorar los resultados educativos provienen desde una concepción constructivista, que sea capaz de fundarse en los saberes propios del que aprende, es decir, el conocimiento que posee el aprendiz y su uso para la construcción del conocimiento matemático (Godino y Batanero, 2016).

Una ruta didáctica y conceptual para generar aproximaciones entre el saber matemático del estudiante y el resultado esperado es mediante la modelación matemática, que se convierte metafóricamente en un puente entre saberes (Frejd \& Bergsten, 2018), lo que permite reconocer el conocimiento matemático por aprender y el uso que el alumno puede hacer de este en la sociedad.

Por otro lado, existen múltiples maneras de conceptualizar la modelación matemática. Los trabajos de Arrieta y Díaz (2016), Schukajlow, Kaiser y Stillamn 
(2018), Stillman, Blum y Kaiser (2017) han logrado convertirse en grandes compendios en este campo de investigación, incluso, dejan entrever las distinciones entre las concepciones, relaciones, objetivos y/o alcances, a fin de enriquecer la práctica disciplinar en la didáctica de la matemática.

Sin embargo, es posible reconocer un foco en común cuando se refiere a modelación o modelación matemática: las acepciones tradicionales plantean el estudio de las relaciones entre la realidad y las matemáticas.

En ese sentido, el modelar concibe que el estrechar tal distancia, permitirá establecer propuestas didácticas con respecto a ciertos objetivos. En nuestro caso, el centro de la práctica de modelación matemática estará direccionado hacia el aprendizaje de la Distribución Normal (DNor). Para lo anterior, se propone un orden desde el planteamiento del estado del arte de la DNor y las preguntas que guían la investigación.

A continuación, se describe a la ingeniería didáctica como diseño metodológico (Artigue, 1995). Para ello, se considera -a partir de una dimensión históricoepistemológica- un análisis de la DNor en el siglo XVI, a fin de reconocer al objeto matemático desde una aproximación no probabilística, la cual se evidencia en los actuales usos del conocimiento matemático en la medicina, en donde el promedio es considerado como la medida de tendencia central en el trabajo matemático del saturador de oxígeno. Además, se presenta el marco conceptual de la modelación matemática, desde una dimensión didáctico-cognitiva. Asimismo, se describe la tarea de modelación, su análisis a priori, la experimentación y el análisis a posteriori, para, finalmente, exponer los resultados y las conclusiones de este trabajo.

\section{Algunos tratamientos didácticos de la DNor}

El tratamiento didáctico que propone el currículum chileno con respecto a la DNor proviene de la esquematización de procesos, a partir de la teoría de la probabilidad (Mineduc, 2015, p.138). Se reconoce un tratamiento y tipología de estrategias, las cuales son intensamente entrenadas para que el estudiante logre un correcto y eficiente uso de las herramientas matemáticas en el análisis de datos, tendiendo, en general, hacia un análisis cuantitativo de variables cuantitativas, y provocando inherentemente que los contextos promuevan una contextualización inicial del objeto matemático, hacia una única aproximación probabilística. 
Tal aproximación de la DNor presupone un tratamiento clásico, es decir, se inicia con definiciones básicas, caracterizaciones simbólicas y gráficas, algunos ejemplos, y finalmente una ejercitación de los procedimientos (California Department of Education, 2015, p. 2; Consejo de Educación Secundaria de Uruguay, 2010, p. 5; Ministerio de Educación Nacional de Colombia, 2006, p. 89). Además, las estrategias de aprendizaje son reducidas hacia la manipulación de fórmulas y procesos algorítmicos.

Por otro lado, una corriente constructivista propone enfocar el interés en el contexto de los datos, los cuales -además de ser números que necesitan una fórmula- son representaciones de comportamientos a analizar. Por ello, se plantea la necesidad de considerar un relativismo constructivista en el carácter epistémico del objeto, para, así, establecer una amplitud tanto en su significado, como en el reconocimiento de propuestas de aprendizaje.

Según Artigue (1990) la dimensión histórico-epistemológica sustenta el desarrollo investigativo en la didáctica de la matemática, siendo de relevancia para la innovación de propuestas que conlleven a ampliar los procesos de aprendizajes. Al respecto, existen investigaciones que señalan que la DNor nace desde la experimentación y del análisis de datos (Conde, 2015; Estepa y Pino, 2013; Stahl, 2006).

Conde (2015) destaca una esquematización de dos caminos epistemológicos que se pueden datar en la historia: uno se desarrolla desde la probabilidad en contextos de problemas de juego; y otro, desde el análisis del error en mediciones experimentales, especialmente en la astronomía. El primero de esos caminos es reconocido en el aula (Mineduc, 2017, p. 13) y en investigaciones didácticas (Alvarado y Batanero, 2008; Alvarado, Galindo y Retamal, 2013; Batanero, Tauber y Sánchez, 2012; Yáñez y Behar, 2009, entre otros).

Por ello, este estudio analiza el surgimiento del conocimiento humano como una fuente de innovación didáctica para ampliar los procesos de enseñanza, siendo la modelación matemática un marco conceptual para el análisis entre el conocimiento de la realidad del estudiante y el conocimiento matemático. Las siguientes preguntas guiarán la investigación: a) ¿Cómo los estudios históricosepistemológicos asociados a la DNor permiten ampliar alternativas didácticas para el aprendizaje de este objeto matemático? y b) ¿Qué tarea de modelación matemática es posible construir desde el análisis histórico-epistemológico de la DNor para propiciar su aprendizaje en la escuela? 


\section{Diseño metodológico}

Desde el enfoque cualitativo, se considera a la ingeniería didáctica como marco metodológico (Artigue, 1995), pues permitirá reconocer las formas de resolución del diseño de aprendizaje y explorar la experimentación. Además, esta es una teoría validada y utilizada para la didáctica de la matemática (Hodgson, Kuzniak y Lagrange, 2016) con un profundo alcance descriptivo e interpretativo (BiknerAshbash, Knipping y Presmeg, 2016).

La ingeniería didáctica se caracteriza por considerar el análisis desde una perspectiva sistémica, logrando particularizar de manera sincrónica y de carácter constitutivo todo el proceso, a partir del estudio preliminar y el contraste de los datos con los instrumentos. Al respecto, Farfán (1997) considera que la validación de resultados, teniendo como base la confrontación de los análisis que se desarrollan en las fases, es un aspecto relevante en la ingeniería didáctica.

En una primera fase de análisis preliminares, Artigue (1995) estructura el proceso a partir de un estudio epistemológico de los contenidos contemplados en la enseñanza. Asimismo, se analiza: a) la enseñanza desde el curriculum y en textos escolares, junto con sus efectos; $y, b$ ) el conjunto de restricciones que operacionaliza el diseño de aprendizaje, en este caso, el de la tarea de modelación, descrita en el marco conceptual de la modelación matemática esbozado por Huincahue, Borromeo-Ferri y Mena-Lorca (2018a).

En una segunda fase, se realiza el análisis a priori de la tarea de modelación, por lo que se construyen y establecen las posibles respuestas de la tarea, lo que conlleva a constantes procesos de ajustes y análisis. La siguiente etapa es de experimentación, pues se integran el diseño y los instrumentos confeccionados para la recolección de datos.

Posteriormente, en la fase de análisis a posteriori y de confrontación se reconocen las acciones realizadas por los estudiantes, a fin de lograr visualizar los aspectos de relevancia para las preguntas de investigación. Finalmente, se concluye con la extracción de los resultados y las conclusiones. 


\section{Fase preliminar}

\section{Análisis histórico-epistemológico}

De acuerdo con Conde (2015), se reconocen dos aproximaciones de la DNor, denominadas por el autor como el camino de la probabilidad y el camino de la curva de los errores (Gauss, 1857; Placket, 1958; Stahl, 2006), reconociendo distintos hitos en su construcción, significados, usos y diversos momentos de construcción, lo que produce un enriquecimiento en las visiones teóricas y prácticas del objeto matemático.

Por otro lado, la dispersión y la estimación de un valor, a partir de varias mediciones relacionadas con un mismo objeto de estudio, es una actividad que trasciende longitudinalmente las culturas y las épocas. Estepa et al. (2013) mencionan que esta situación la reconocen, inicialmente, en el estudio de los cuerpos celestes desde épocas babilónicas (c.f. 650 a. C.), teniendo como tarea constante crear conocimiento científico o tecnológico para precisar sus mediciones hasta el día de hoy (Stahl, 2006, p. 16). Otro ejemplo de ello, citado por Plackett (1958), es Hipparcus (190-120 a. C.), quien planteó una estimación de la máxima duración de un año en tres cuartos de día, considerando la mitad del rango de sus múltiples observaciones.

En otro orden de ideas, en el siglo XVI, Tycho Brahe (1546-1601) fue pionero en el desarrollo epistemológico de la DNor, al ofrecer una variedad, tanto en el estudio de los cuerpos celestes, como en el estudio de dispersión de datos, siendo uno de los últimos astrónomos en estudiar los cuerpos celestes con el ojo desnudo. La técnica metodológica que utilizó en la astronomía fue la repetición de las medidas (Stahl, 2006), asumiendo que todos los datos poseen errores, por lo que enfocó el análisis no solo en los datos, sino en el comportamiento del error inherente en los datos. En este sentido, Huincahue, Durand, González, Ramírez y Toro (2018b) plantean que este método varía, pues algunos usaban la media, o bien, mediana para determinar un solo valor a partir de un conjunto de datos, pero siempre con el fin de modelar los errores del conjunto de datos y determinar el valor más representativo del conjunto.

Incluso, Galileo Galilei (1564-1642), apoyado en los resultados de Tycho Brahe, realizó un planteamiento formal e institucional del error, realzando este como un elemento en las mediciones astronómicas, y formuló cinco propiedades a las observaciones de fenómenos. Por los intereses propios de la investigación, 
solo nos centraremos en las tres primeras, las cuales describimos a continuación ${ }^{1}$ (Conde 2015, p. 63):

1. Hay solamente un número, el cual da la distancia de la estrella al centro de la tierra, la verdadera distancia.

2. Todas las observaciones están cargadas de errores, debido al observador, los instrumentos y otras condiciones observacionales.

3. Las observaciones son distribuidas simétricamente alrededor del valor verdadero.

Estas afirmaciones posicionan al error en el estudio de mediciones de cuerpos celestes. Además, en su clásica obra Dialogue concerning the two chief world systems-Ptolemaic \& Copernican (Galilei, 1962), el autor plantea que el valor más representativo (aquel que minimiza el error) se asocia a la mediana y no al promedio, siendo de interés para el análisis de datos que realiza y distinguiéndose en este punto de Tycho Brahe.

Posterior a la variedad de enfoque propuesto por Tycho, Thomas Simpson (1710-1761) construye la distribución uniforme discreta (1786) y distribución triangular discreta (1756), considerando metodológicamente la existencia del error en la medición de datos, logrando un convencimiento sobre la media como un valor de gran representatividad. Este autor concluye que, al tomar la media de un número de observaciones, se disminuye de gran manera las posibilidades de los errores (Estepa et al., 2013).

Por otro lado, y gracias a la similar época con Simpson, Daniel Bernoulli (17001782) plantea que, a partir de sus múltiples experimentaciones, "el verdadero valor" está en el centro de la curva circular de la distribución, estableciendo, en palabras de Stahl (2006), que: "...las observaciones son sumadas entre sí y la suma dividida por el número de observaciones: el cociente es entonces aceptado como el verdadero valor de la cantidad requerida, hasta que se tenga mejor información y más cierta..." (p. 104).

Las 5 propiedades son formuladas en Galilei (1962). Versiones modernas de las 5 propiedades son posibles de encontrar en Hald (1998) o Stahl (2006), por ejemplo. 
Otro autor contemporáneo de T. Simpson y D. Bernouilli, que realizó contribuciones al estudio del error, fue Jean-Henri Lambert (1728-1777). Según el mencionado investigador, la media aritmética no difiere del valor verdadero, por lo que concluye que existen diferentes tipos de errores en las mediciones, por lo cual propone una primitiva medida de fiabilidad para la media (Estepa et al., 2013). Gauss (1777-1855), por medio de las observaciones astronómicas y habiendo estudiado los avances de la Teoría de Error en la época, también construyó modelos para la predicción de la posición y órbita de un cuerpo celeste, utilizando el criterio de mínimos cuadrados (Conde, 2015; Stahl, 2006). Además, Gauss en su libro Theoria motus corporum coelestium in sectionibus conicis solem ambientium, afirma:

"Se ha considerado ciertamente como un axioma la hipótesis de que si alguna cantidad ha sido determinada por varias observaciones directas, realizadas en las mismas circunstancias y con igual cuidado, la media aritmética de los valores observados proporciona el valor más probable" (Gauss, 1857, p. 258).

Gauss, sobre la base de las afirmaciones, demostró la simetría, diferenciabilidad y unicidad de un máximo local y concluyó que la densidad de la curva del error está determinada por la siguiente función:

$$
\varphi(x)=\frac{\boldsymbol{h}}{\sqrt{\pi}} e^{-h^{2} x^{2}}
$$

Donde $\mathrm{h}$ representa la medida de precisión de las observaciones y $\mathrm{x}$ es el error.

Posteriormente, Karl Pearson (1855-1936) propuso el término de normal a la curva del error, como curva normal (Stahl, 2006). Tal compendio describe una línea de tiempo mostrada en la Figura 1.

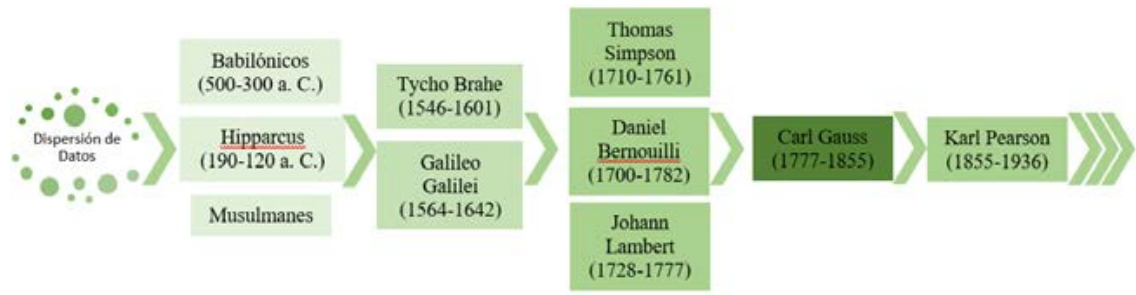

Figura 1. Evolución de la curva del error en la DNor. 
El contexto presentado anteriormente define un tratamiento a partir del análisis del error en el manejo de datos, que caracteriza a la DNor como un modelo descriptivo, considerando repetidamente la medición de un fenómeno, lo cual fue incipientemente tratado desde la cultura babilónica, aplicado por primera vez en los trabajos de Tycho Brahe (Huincahue et al., 2018b) y planteado formalmente por Gauss al señalar que: el promedio es la medida de tendencia central adecuada para un comportamiento normal de datos. Todas estas contribuciones son consideradas hitos epistemológicos con respecto a la construcción de la DNor, específicamente motivado desde el estudio del error en mediciones astronómicas.

Este constructo histórico-epistemológico no solo ha estado en un entorno conceptual y matemático, sino que también prevalece en forma y uso en distintas comunidades disciplinares, como, por ejemplo, en herramientas de medición específicas de la medicina.

La medicina: mediciones y herramientas

La medicina realiza múltiples análisis estadísticos de tipo clínico de diversa índole. Solo a modo de ejemplo, tales estudios ayudan en la determinación de parámetros de normalidad, lo que para San Pedro, Grandi, Larguía y Solana (2001) se traduce en un apoyo para la toma de decisiones de la salud de las personas, específicamente, en determinar diagnósticos epidemiológicos que se aproximen al impacto de enfermedades en recién nacidos.

En general, la mayoría de los análisis buscan la normalidad dentro de la variabilidad de un grupo de datos (interpretación de gráficas, niveles de curtosis, etc.). En particular -en la medicina-, los análisis clínicos se realizan con diferentes artefactos. Uno de esos equipos es el saturómetro, una peculiar instrumentalización en la toma de datos. A continuación, se realizará una descripción de su función, utilidad, funcionamiento, qué fenómenos registra y cómo entrega el resultado.

\section{El saturómetro}

Este es un artefacto que utiliza la técnica radial de la pulsioximetría, que mide dos fenómenos según el Manual de oximetría de pulso global de la Organización Mundial de la Salud [OMS] (2010). 
El primer fenómeno a medir es la saturación de oxígeno de la hemoglobina en sangre arterial: en primera instancia se habla de que los glóbulos rojos tienen hemoglobina, que es una molécula que transporta oxígeno. Cada vez que la hemoglobina pasa por los pulmones, puede albergar hasta cuatro moléculas de oxígeno. Entonces, si acaso todas las hemoglobinas están transportando oxígeno en cada lugar de unión, se establece que la saturación de oxígeno en sangre arterial es del $100 \%$ (OMS, 2010).

El saturómetro registra la lectura de la saturación de oxígeno, según Casado y Seco (2010), por medio de dos longitudes de onda de luz, una de $660 \mathrm{~nm}$ (roja) y otra de $940 \mathrm{~nm}$ (infrarroja), que son emitidas por los diodos emisores de luz, las cuales son absorbidas por los tejidos conectivos (como son la piel, los huesos y la sangre), produciendo una variación del color según la saturación de la hemoglobina. Luego, el foto-detector detecta la luz transmitida y el microprocesador transforma esta información en el valor de la saturación de oxígeno (OMS, 2010). Posteriormente, las lecturas realizadas por el saturómetro son interpretadas clínicamente como se muestra en la Tabla 1.

\section{Tabla 1}

Interpretación clínica de la saturación de oxígeno.

\begin{tabular}{cl}
\hline$\%$ Saturación & \multicolumn{1}{c}{ Acción } \\
\hline$>95 \%$ & No actuación inmediata. \\
\hline $95-90 \%$ & $\begin{array}{l}\text { Tratamiento inmediato y monitorización de la respuesta al mismo. } \\
\text { Según la respuesta, valorar derivación al hospital. Los pacientes } \\
\text { con enfermedades respiratoria crónica toleran bien saturaciones en } \\
\text { torno a estos valores. }\end{array}$ \\
\hline$<90 \%$ & $\begin{array}{l}\text { Enfermo grave. Hipoxia severa. Oxigenoterapia + Tratamiento y } \\
\text { traslado al Hospital. }\end{array}$ \\
\hline$<80 \%$ & Valorar intubación y ventilación mecánica. \\
\hline
\end{tabular}

Fuente: Casado y Seco (2010).

El segundo fenómeno es la frecuencia cardiaca, que es la cantidad de latido por minuto, promediado cada 5 a 20 segundos. El saturómetro lo detecta por medio de un flujo pulsátil a través de un sensor, permitiendo conocer si son normales 0 no los latidos del corazón. En relación a la frecuencia cardiaca de los niños, esta tiende a ser más alta que en los adultos: los niños recién nacidos hasta los dos años tienen una frecuencia cardiaca normal entre los 100 y 180; los niños de dos a 
diez años poseen una frecuencia cardíaca normal entre 60 y 140; y desde los diez años en adelante, el rango de normalidad es entre los 50 y los 100.

Es importante destacar que, se debe considerar la frecuencia cardiaca para realizar una buena lectura de los datos, debido a su influencia en la curva pletismográfica de la señal de oximetría, la cual se identifica como señal normal en la Figura 2.

La pantalla del saturómetro muestra los datos de: la saturación de oxígeno, la frecuencia cardiaca y la curva pletismográfica de la señal de oximetría.

\section{Señal normal}
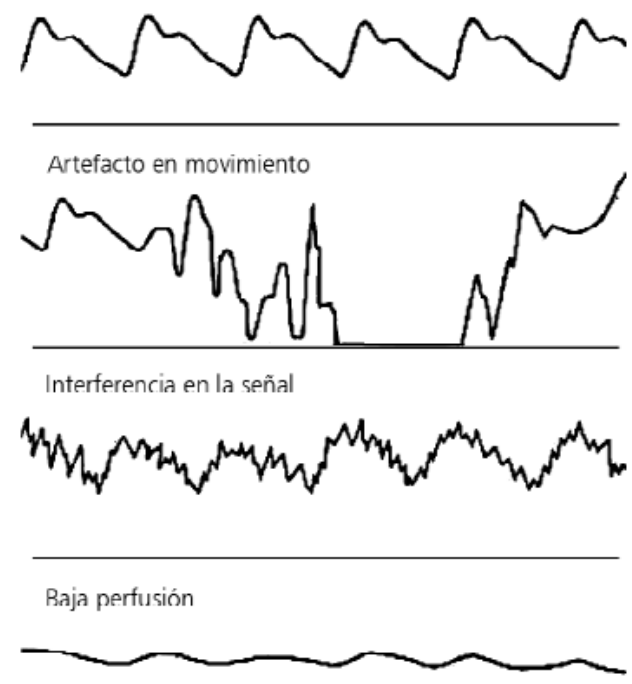

Figura 2. Patrones de curva pletismográfica de la señal de oximetría.

Fuente: Palacios, Álvarez, Schönffeldt, Céspedes, Gutiérrez y Oyarzún (2010). 
Esta técnica radial, sustentada por el análisis epistemológico, se utilizará con el fin de obtener información de un determinado grupo de estudiantes. Luego, por medio de la recolección de datos y el análisis estadístico, se construirá un acercamiento al concepto de la DNor. Sin embargo, se requiere una aproximación didáctica, tanto para la confección de instrumentos, como para el análisis de los datos, el cual será vía modelación matemática.

\section{Análisis didáctico}

En Chile, el modelar es considerado como una habilidad en las bases curriculares que tiene por objetivo "...construir una versión simplificada y abstracta de un sistema que opera en la realidad" (Mineduc, 2016, p. 106), por ello adquiere un carácter transversal e integrador del conocimiento matemático (Huincahue et al., 2018a). Por eso, es que se plantea un constructo teórico pertinente para que los estudiantes sean responsables de su propia construcción del conocimiento matemático.

William and Goos (2013) destacan que los enfoques tradicionales de modelación matemática describen su actividad a partir de ciclos de modelación que relacionan la realidad y las matemáticas, siendo estos entes que ideológicamente se reconocen distanciados. De tales enfoques, uno que ha influenciado los currículos latinoamericanos, norteamericanos y europeos, es el ciclo de Blum (Blum y Lei $\beta, 2007$ ). Sin embargo, tal ciclo posee una aproximación cognitiva, que es comúnmente denominada como el ciclo de Blum-Borromeo (Borromeo-Ferri, 2010).

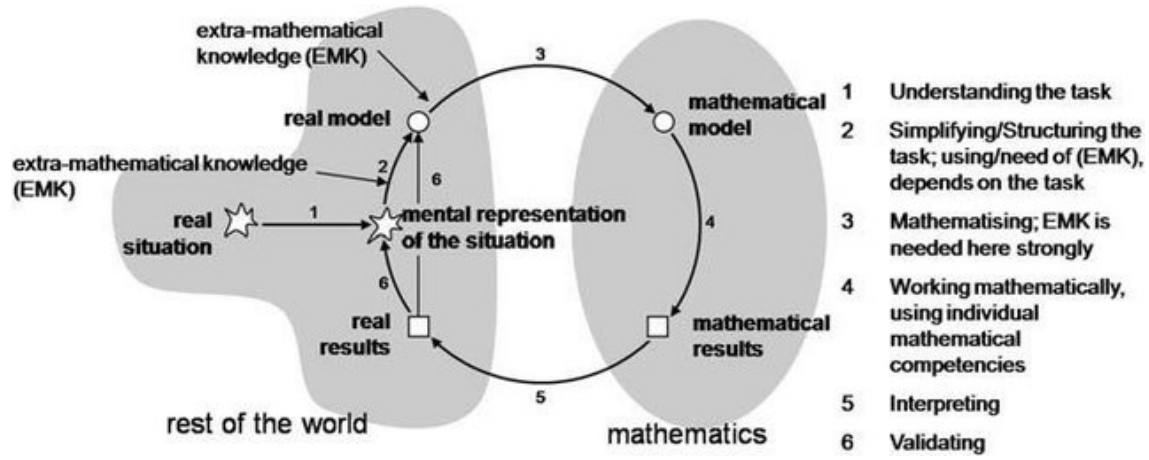

Figura 3. Ciclo de modelación Blum-Borromeo.

Fuente: Borromeo-Ferri (2010). 
El ciclo de modelación se inicia con una situación real, que en nuestro caso es representada por el fenómeno de la saturación de oxígeno en sangre. A continuación, viene un entendimiento de la tarea de modelación para crear una representación mental de los datos arrojados por el saturómetro, focalizando y simplificando los datos. Posteriormente, se llega a un modelo real, producto de una nueva simplificación del problema a nivel consciente del estudiante. Seguidamente, existe un proceso de matematización para obtener un modelo matemático muy influenciado por el conocimiento extramatemático y que, al momento de intervenir las competencias matemáticas, produce un resultado matemático. Este resultado está determinado por la influencia de los elementos de la estadística, ya sea a través de la representación tabular, representación gráfica, moda, media 0 mediana. Finalmente, se obtienen resultados matemáticos e interpretaciones de los datos recogidos, que demuestran con resultados reales el fenómeno de la saturación de oxígeno en sangre, validándose en la representación de la situación en el mundo real (Blum, 2015).

Por otro lado, las tareas de modelación matemática pueden poseer múltiples características y ser clasificadas según objetivos, alcances e intereses educativos o investigativos (Araújo, 2009). Ese fenómeno es estudiado por Kaiser y Sriraman (2006), quienes esbozan una clasificación de perspectivas de modelación: realista, contextual, educacional, sociocrítica, epistemológica o cognitiva. Las perspectivas en una tarea de modelación no son excluyentes con respecto a las demás, es decir, puede existir una perspectiva predominante, pero, además, tener varias perspectivas de forma simultánea.

Dada la primera pregunta de investigación, se privilegiará una teorización de la DNor a partir de los antecedentes histórico-epistemológicos, por lo tanto, la tarea estará inclinada hacia la perspectiva epistemológica, ${ }^{2}$ la cual privilegia el entendimiento y la descripción de la naturaleza de las actividades matemáticas, respetando tanto su surgimiento como su construcción epistémica. En esta perspectiva, existe un centro en el desarrollo teórico de la propuesta, que trasciende en importancia a la tarea misma (Huincahue, 2017, p. 63); esto no significa que el principio realista no exista, sino que no es la característica que se pretende privilegiar en la tarea. La característica a privilegiar en la perspectiva epistemológica es el conocimiento subyacente de la tarea.

2 Tanto en Kaiser et al. (2006) como en Huincahue (2017) se realiza un desglose y caracterización de cada perspectiva. 
En esta segunda fase se deben tomar decisiones sobre las variables de la investigación. Estas variables de comando nacen desde el análisis preliminar, caracterizado por el análisis a la enseñanza tradicional y sus efectos, las concepciones epistemológicas de la DNor, lo cognitivo que rodea al concepto y lo didáctico asociado al sistema de enseñanza. Con base en lo anterior, se realiza una organización de una tarea de modelación.

La confección de la tarea (ver Anexo 1) fue orientada bajo el camino epistemológico de la curva de errores, proyectada para un trabajo grupal de tres personas y construida en tres momentos: a) descripción del manejo del saturómetro e interpretación de datos; b) recolección de datos con el saturómetro y su respectivo ingreso al software GeoGebra; y c) análisis de datos y resultados.

\section{Fase de análisis a priori}

El análisis a priori es concebido -en términos de Artigue (1995) -, como un análisis de control de significados, es decir, las posibles respuestas y errores que se presentarán al momento de la implementación de la tarea de modelación. Parte del extenso análisis realizado es descrito en la Tabla 2. 


\section{Tabla 2}

Algunas respuestas del análisis a priori.

\begin{tabular}{|c|c|}
\hline \multirow[t]{9}{*}{ Estrategias } & $\begin{array}{l}\text { Los estudiantes construyen una tabla de frecuencia, donde sintetizan } \\
\text { los datos recogidos de la saturación de oxígeno. }\end{array}$ \\
\hline & Se ingresan los datos a la hoja de cálculo de GeoGebra. \\
\hline & Grafican los datos recogidos, desde la tabla de frecuencias. \\
\hline & Escogen a uno de sus pares para ser el sujeto de estudio. \\
\hline & Se escoge un operador del saturómetro. \\
\hline & Se designa un asistente, encargado de digitar y registrar los datos. \\
\hline & $\begin{array}{l}\text { Considerar el valor de la moda como saturación de oxígeno } \\
\text { referencial. }\end{array}$ \\
\hline & Calcular la media aritmética de datos agrupados, de forma manual. \\
\hline & Calcular la media de datos agrupados, utilizando GeoGebra. \\
\hline \multirow[t]{6}{*}{ Pregunta 1} & Se considera la moda como la saturación de oxígeno del compañero \\
\hline & Se considera la media como la saturación de oxígeno del compañero \\
\hline & La saturación de oxígeno de Javiera es 97 (aproximadamente) \\
\hline & La saturación de oxígeno de Javiera es 96,72 \\
\hline & En ambas coinciden la media y la moda \\
\hline & $\begin{array}{l}\text { El número que tiene mayor frecuencia es el representante de la } \\
\text { moda y la media, en ambos casos }\end{array}$ \\
\hline \multirow[t]{8}{*}{$\begin{array}{l}\text { Errores } \\
\text { Pregunta } 1\end{array}$} & $\begin{array}{l}\text { No considerar el número con más frecuencia como número } \\
\text { referencial }\end{array}$ \\
\hline & $\begin{array}{l}\text { Calcular el promedio de los valores de las variables, sin considerar } \\
\text { su frecuencia }\end{array}$ \\
\hline & Calcular la media aritmética como si fueran datos no agrupados \\
\hline & Confundir el cálculo de la media y de la mediana \\
\hline & $\begin{array}{l}\text { Considerar como saturación de oxígeno del compañero la media } \\
\text { aritmética de datos no agrupados }\end{array}$ \\
\hline & La moda y la media son diferentes \\
\hline & No hay relación entre los resultados de Javiera y el compañero \\
\hline & Por causa de los errores anteriores, se declara que son diferentes \\
\hline
\end{tabular}




\section{Fase de experimentación}

La tarea de modelación fue implementada en un colegio ubicado en la ciudad de Rancagua, Región del Libertador General Bernardo O'Higgins y en el nivel de cuarto medio científico-humanista. Los estudiantes fueron elegidos al azar y sus edades estaban comprendidas entre 17 y 18 años; de ellos, 3 eran mujeres y 6 hombres. Asimismo, se conformaron tres grupos, que realizaron la tarea en 90 minutos, resolviendo cada uno de los ítems propuestos.

Los alumnos escucharon las instrucciones sobre el desarrollo de la actividad, designaron roles según las necesidades de la tarea y aprendieron a utilizar el saturómetro, específicamente, focalizándose en la curva pletismográfica. Luego, los estudiantes recolectaron los 50 datos de un sujeto de prueba y escribieron los resultados en una tabla realizada por el grupo de investigación. Al respecto, en general, hubo una correcta realización de la tarea en cada uno de los momentos y los tres grupos terminaron la totalidad de la tarea.

\section{Fase de análisis a posteriori y confrontación}

Para el análisis a posteriori (Artigue, 1995), se presentaron inicialmente las gráficas que los estudiantes realizaron. Allí, se resumieron las diferentes mediciones sobre el fenómeno de saturación de oxígeno en la sangre en el sujeto de estudio (un estudiante por grupo).

\section{Tabla 3}

Gráficas asociadas a cada grupo.

$\frac{\text { G1 }}{0}$

Luego de realizar la organización de los datos y confección de las diferentes gráficas, los grupos contestaron las preguntas de la tarea. Las respuestas asociadas a cada pregunta se presentan en Tabla 4. 


\section{Tabla 4}

Respuestas a las preguntas de modelación por cada grupo.

\begin{tabular}{|c|c|c|}
\hline Preguntas & Grupos & Respuestas \\
\hline \multirow[t]{3}{*}{ a } & 1 & $\begin{array}{l}\text { 97, porque es el número más frecuente en cuanto a cantidad } \\
\text { de apariciones en los datos obtenidos. }\end{array}$ \\
\hline & 2 & $\begin{array}{l}>95 \% \text {, estable, ya que su promedio es de } 98,38, \text { por } 10 \\
\text { tanto, no requiere atención. }\end{array}$ \\
\hline & 3 & $\begin{array}{l}\text { La saturación de oxígeno referencial es } 97 \text { porque de ser la } \\
\text { que más se repite, es una variación normal en las personas. }\end{array}$ \\
\hline \multirow[t]{3}{*}{ b } & 1 & La media es 97. \\
\hline & 2 & 98,38 \\
\hline & 3 & media $=96,6$. \\
\hline \multirow[t]{3}{*}{ c } & 1 & $\begin{array}{l}\text { Basándonos en la tabla, se podría decir que predomina el } \\
97, \text { ya que sería la forma normal del individuo y } 93 \text { sería el } \\
\text { menor valor sacado, por conclusión que esa frecuencia es } \\
\text { la que menor vez se ve y dentro de lo normal estaría entre } \\
93 \text { y } 97 \text { de frecuencia. }\end{array}$ \\
\hline & 2 & $\begin{array}{l}\text { Sería aprox. } 98 \% \text {, ya que es } 98,38 \text { y según la tabla en un } \\
>95 \% \text {, por lo tanto, no necesita acción médica. }\end{array}$ \\
\hline & 3 & $\begin{array}{l}\text { Según las consideraciones la saturación de oxígeno en } \\
\text { condiciones normales es } 97 \text {, estable variando entre eso y } \\
\text { un poco más, dependiendo de la situación y el control de la } \\
\text { respiración es como va cambiando el ingreso de oxígeno. }\end{array}$ \\
\hline \multirow[t]{3}{*}{ d } & 1 & $\begin{array}{l}\text { En comparación con la tabla anterior, observamos que son } \\
\text { literalmente iguales y se podría decir que los dos están en un } \\
\text { rango de la normalidad que sería } 93>97 \text { y podríamos decir } \\
\text { que son sanos y hasta con la condición de un deportista. }\end{array}$ \\
\hline & 2 & $\begin{array}{l}\text { La saturación de oxígeno de Javiera varía más que mi } \\
\text { compañero, por lo que tiene una desviación más amplia y } \\
\text { se parece en que ambos están }>95 \% \text {. }\end{array}$ \\
\hline & 3 & $\begin{array}{l}\text { La saturación de oxígeno de oxígeno de Javiera es } 97 \text {, a } \\
\text { comparación con nuestro primer compañero la saturación } \\
\text { de ambos es muy similar, ya que ambos obtuvieron la } \\
\text { misma saturación de oxígeno, pero, a su vez, Javiera tenía } \\
\text { datos un poco más variados, lo que en su medía la S. de } 0 \text {. } \\
\text { varió en } 1 \text { decimal. }\end{array}$ \\
\hline
\end{tabular}


Todos los grupos calcularon de forma satisfactoria el promedio de los datos. Solo se diferencian en que unos lo aproximaron y otros no. Una gran ayuda en este cálculo fue que los estudiantes buscaron cómo calcular la media aritmética de datos agrupados o buscaban en el software el cálculo automático de esta medida de tendencia central.

\section{Resultados}

Al realizar el estudio histórico-epistemológico de la DNor, surgieron elementos que dieron un lineamiento para la construcción de la tarea de modelación, de tal manera que se produjo una sistematización en la construcción matemática del concepto a través del ciclo de Blum-Borromeo (Borromeo-Ferri, 2010).

Los estudiantes, durante la recolección de los datos, reconocieron ciertas regularidades. Los tres grupos aludieron estas regularidades al valor más frecuente y al valor promedio, cuyas caracterizaciones se reconocen en el análisis histórico-epistemológico de la DNor. Estos datos fueron el centro de discusión que permitió desarrollar y contestar a las diferentes preguntas, traduciendo los resultados matemáticos en la realidad, por ejemplo, en la respuesta a la pregunta "a" de G2 y G3.

A continuación, cada grupo realizó la interpretación de los datos desde el resultado matemático al resultado real, utilizando la Tabla 1, la cual les proporcionó la información necesaria y suficiente para determinar el grado de normalidad del resultado matemático. Todo este proceso de confección de un modelo matemático (el resultado, su interpretación al resultado real y todas las fases del modelo) fueron reconocidas en los grupos, cuyas respuestas estaban en concordancia con el análisis histórico-epistemológico y la realidad, que en este caso era la medicina.

El caso del sujeto experimental 1 (G1) fue analizado paralelamente por su estilo de vida. Según su grupo, tiene una saturación de oxígeno en la sangre de $97 \%$, la cual, según la interpretación de los datos, es un valor dentro de la normalidad (los estudiantes validaron con la media aritmética). El grupo declara: "Es deportista, pues juega fútbol, esto es un factor que influye en las lecturas de la saturación de oxígeno". Esto se debe a que en la última pregunta, sus compañeros declaran que $\mathrm{C} 1$ tiene esa saturación de oxígeno normal, por causa de que es sano y deportista (ver respuesta a la pregunta $d$ de $\mathrm{G} 1$ ). 
El grupo de $\mathrm{C} 1$ determinó ese valor verdadero de la saturación de oxígeno en el promedio de los datos recogidos, además, logró una gráfica similar a la clásica campana gaussiana (ver Tabla 3, G1), situación que ayudó a los estudiantes a realizar un prolijo análisis de las preguntas sobre el "verdadero" valor.

Los demás grupos también desarrollaron sus análisis normalmente, pero sus gráficos y datos, están sujetos a estudios sobre los factores que influyeron en sus resultados, porque, según Estepa et al. (2013), existen diferentes tipos de fuentes de dispersión de los datos, en este caso dispersión en la medida. Además, $\operatorname{con}^{n}=50$ (ver Anexo 1) se espera una mayor dispersión.

\section{Conclusiones y discusión}

El estudio histórico-epistemológico realizado y las características que surgieron de este ayudaron a una confección de la tarea de modelación, guiada por el constructo epistemológico investigado. Además, se evidenció que es posible ampliar el enfoque conceptual del objeto matemático que impera en los estudios de la didáctica de la matemática.

La tarea propició que los grupos reconocieran al valor más frecuente como la media aritmética de los datos y se logró operacionalizar la resolución de la tarea para favorecer el conocimiento emergente, reconociendo construcciones de la DNor a partir de la aproximación enunciada.

La utilización del instrumento médico (saturómetro) como artefacto en la tarea de modelación es una ayuda para realizar la conexión entre lo cotidiano y lo matemático, pues el aprendizaje desde elementos situados ayuda y propicia el conocimiento en los estudiantes y que aquello que han construido tenga significado. Igualmente, así como en la medicina, se evidencian usos de la DNor en estudios de otras disciplinas y en otros usos, que pueden ayudar a construir tareas de modelación y propiciar el aprendizaje desde elementos contextualizados.

Desde una aproximación conceptual, el análisis realizado evidencia contribuciones presentes en la Figura 1, que permiten colaborar con la investigación histórica o epistemológica de la DNor (Conde, 2015).

La primera propuesta, anteriormente en Huincahue et al. (2018b), es reconocer a Tycho Brahe y su estudio como uno de los precursores en el análisis de la 
medición de cuerpos celestes; la segunda, hace alusión a Karl Pearson, quien acuñó el concepto de DNor como una curva que muestra la normalidad.

Finalmente, esta investigación, que nació desde un contexto escolar, buscó fortalecer la comunidad de la didáctica de la matemática, al proporcionar insumos para futuras investigaciones, al considerar que cada esfuerzo realizado produce mejoras en las metodologías de la enseñanza y aprendizaje de objetos matemáticos. Además, estos insumos no solo quedan en la investigación, sino que forman parte de una red de apoyo para aquellos docentes que realizan clase de DNor en cuarto medio, por lo que podrán mejorar sus prácticas docentes.

\section{REFERENCIAS BIBLIOGRÁFICAS}

ALVARADO, H. Y BATANERO, C. (2008). "Meaning of Central Limit Theorem in university statistics and probability textbooks". Estudios Pedagógicos, 34(2), 7-28.

ALVARADO, H.; GALINDO, M. Y RETAMAL, L. (2013). "Comprensión de la distribución muestral mediante configuraciones didácticas y su implicación en la inferencia estadística". Enseñanza de las Ciencias, 31(2), 75-91.

ARAÚJO, J. (2009). "Uma abordagem sócio-Crítica da modelagem matemática: a perspectiva da educação matemática crítica". Alexandria. Revista de Educação em Ciência e Tecnologia, 2(2), 55-68. Recuperado de: https://periodicos.ufsc.br/ index.php/alexandria/article/view/37948/28976

ARRIETA, J. Y DÍAZ, L. (Eds) (2016). Investigaciones latinoamericanas en modelación. Matemática Educativa. Ciudad de México: Gedisa.

ARTIGUE, M. (1990). "Epistémologie et didactique". Reserches en didactique des mathématiques. 10(23). París.

ARTIGUE, M. (1995). "Ingeniería didáctica" en Pedro Goméz (Ed.). Ingeniería didáctica en educación matemática, 33-59. Bogotá: Una empresa docente.

BATANERO, C.; TAUBER, L., \& SÁNCHEZ, V. (2001). "Significado y comprensión de la distribución normal en un curso introductorio de análisis de datos". Quadrante, 10(1), 59-92. 
BIKNER-ASHBASH, A; KNIPPING, CH. Y PRESMEG, N. (Eds.) (2016). Approaches to qualitative research in mathematics education. Londres: Springer.

BLUM, W. \& LEIß, D. (2007). "'Filling Up' - the problem of independence-preserving teacher interventions in lessons with demanding modelling tasks" en Bosch, Marianna (Ed.): CERME 4 - Proceedings of the Fourth Congress of the European Society for Research in Mathematics Education, 1623-1633.

BLUM, W. (2015). "Quality teaching of mathematical modelling: What do we know, what can we do?" in S. J. Cho (Ed.). The Proceeding of the 12th International Congress on Mathematical Education, 73-96. Dodrecht: Springer.

BORROMEO-FERRI, R. (2010). "On the influence of mathematical thinking styles on learners' modeling behavior". Journal für Mathematik-Didaktik, 31(1), 99-118.

CALIFORNIA DEPARTMENT OF EDUCATION (2015). Advanced placement probability and statics chapter of the mathematics framework for California public schools: Kindergarten Through Grade Twelve. Sacramento: California Department of Educatión. Recuperado de: https://www.cde.ca.gov/ci/ma/cf/mathfwchapters. asp.

CASADO, M. Y SECO, A. (2010). Técnicas en AP: Pulsioximetría. La Coruña. España.

CONSEJO DE EDUCACIÓN SECUNDARIA (2010). Programa de Matemática Núcleo Común, Segundo de Bachillerato. Recuperado de: https://www.ces.edu. uy/index.php/propuesta-educativa/20249.

CONDE, A. (2015). "La distribución normal una rápida revisión histórica". Heurística, 17, 59-65.

ESTEPA, A. Y PINO, J. (2013). "Elementos de interés en la investigación didáctica y enseñanza de la dispersión estadística". Números. Revista de Didáctica de las Matemáticas, 83, 43-63.

FARFÁN, R. M. (1997). Ingeniería Didáctica: Un estudio de la variación y el cambio. México DF: Grupo Editorial Iberoamericana. 
FREJD, P. \& BERGSTEN, C. (2018). "Professional Modellers' conceptions of the notion of mathematical modelling: ideas for education". ZDM - The International Journal on Mathematics Education. 50(1-2), 117-127.

GAUSS, C. (1857). Theory of motion of the heavenly bodies moving about the sun in conic section. (Trans. C. Davis). Boston: Little, Brown and Company (obra original publicada en 1809).

GALILEI, G. (1962). Dialogue concerning the two chief world systems-Ptolemaic \& Copernican (traducción de S. Drake), $2^{\circ}$ ed. Berkeley: Univ. California Press.

GODINO, J. Y BATANERO, C. (2016). "Implicaciones de las relaciones entre epistemología e instrucción matemática para el desarrollo curricular: el caso de la Combinatoria". La matematica e la suadidattica, 24(1-2), 17-39. Recuperado de: http://enfoqueontosemiotico.ugr.es/documentos/Godino_Batanero_LaMate_ SuaDida_2016_Epistemologia_instruccion.pdf

HALD, A. (1998). A history of mathematical statistics. From 1750 to 1930. New York: John Wiley \& Son Inc.

HUINCAHUE, J. (2017). Propuesta de modelación matemática en la formación de profesores y bases para una variedad de modelación desde la Teoría Socioepistemológica. Valparaíso, Chile: Pontificia Universidad Católica de Valparaíso. Recuperada de: http://opac.pucv.cl/pucv_txt/txt-2500/UCC2902_01. pdf

HUINCAHUE, J.; BORROMEO-FERRI, R. Y MENA-LORCA, J. J. F. (2018a). "EI conocimiento de la modelación matemática desde la reflexión en la formación inicial de profesores de matemática". Enseñanza de las ciencias, 36(1), 99115. Recuperado de: https://ddd.uab.cat/pub/edlc/edlc_a2018v36n1/edlc_ a2018v36n1p99.pdf

HUINCAHUE, J.; DURAND-GALAZ, B.; GONZÁLEZ CAMUS, C; RAMÍREZALBORNOZ, C. Y TORO ITURRIETA, A. (2018b). "La distribución normal como un modelo de los errores: análisis desde la teoría de situaciones didácticas" en Del Valle, T. y Henríquez-Rivas, C. (Comp.). Investigaciones en la formación inicial del profesor de matemáticas. Trabajo de estudiantes tesistas, 53-70. Santiago, Chile. Ediciones UCSH. 
HODGSON, B.; KUZNIAK, A. Y LAGRANGE, J. (Eds.) (2016). The didactics of mathematics: Approaches and issues a homage to Michéle Artigue. Paris: Springer.

KAISER, G. Y SRIRAMAN, B. (2006). "A global survey of international perspectives on modeling in mathematics education". ZDM - The International Journal on Mathematics Education, 38(3), 302-310.

MINEDUC (2015). Matemática. Programa de estudio cuarto año medio. Santiago de Chile: Mineduc.

MINEDUC (2016). Bases Curriculares $7^{\circ}$ básico a $2^{\circ}$ medio. Santiago de Chile: Mineduc.

MINEDUC (2017). Experiencias de aprendizaje: Matemáticas. Santiago: Maval S.A. Recuperado de: https://media.mineduc.cl/wp-content/uploads/sites/28/2017/11/ Matem\%C3\%A1tica-4\%C2\%BA-Medio.pdf

MINISTERIO DE EDUCACIÓN NACIONAL. (2006). Estándares básicos de competencias en matemáticas. Bogotá: Ministerio de Educación Nacional. Recuperado de: https://www.mineducacion.gov.co/cvn/1665/article-116042.html

ORGANIZACIÓN MUNDIAL DE LA SALUD. (2010). Manual de Oximetría de Pulso Global. Ginebra: Organización Mundial de la Salud. Recuperado de: http:// www.lifebox.org/wp-content/uploads/WHO-Pulse-Oximetry-Training-ManualFinal-Spanish.pdf

PALACIOS, S.; ÁLVAREZ, C.; SCHÖNFFELDT, P.; CÉSPEDES, J.; GUTIÉRREZ, M. Y OYARZÚN, M. (2010). "Guía para realizar oximetría de pulso en la práctica clínica". Revista Chilena de Enfermedades Respiratorias, 26(1), 49-51.

PLACKET, R. L. (1958). "The principle of the arithmetic mean" en E. S. Pearson y M. Kendall (1978). Studies in the History of Statistics and Probability. London: Charles Griffin.

SAN PEDRO, M.; GRANDI, C.; LARGUÍA, M., Y SOLANA, C. (2001). "Estándar de peso para la edad gestacional en 55706 recién nacidos sanos de una maternidad pública de Buenos Aires". Medicina, 61(1), 15-22. 
SCHUKAJLOW, S.; KAISER, G. \& STILLMAN, G. (2018). "Empirical research on teaching and learning of mathematical modelling: a survey on the current state-ofthe-art". ZDM - The International Journal on Mathematics Education. 50(5), 5-18.

STAHL, S. (2006). The evolution of the Normal Distribution. Mathematics Magazine, 79(2), 96-113.

STILLMAN, G. A.; BLUM, W., \& KAISER, G. (Eds.). (2017). "Mathematical modelling and applications: Crossing and researching boundaries in mathematics education" in G. Kaiser \& G. A. Stillman (Eds.), International perspectives on the teaching and learning of mathematical modelling. New York, NY: Springer.

WILLIAMS, J. \& GOOS, M. (2013). Modelling with mathematics and technologies. In M. A. Clements, A. J.; Bishop, C.; Keitel, J.; Kilpatrick \& F. K. S. Leung (Eds.). Third international handbook of mathematics education, 549-569. Berlin: Springer.

YÁNEZ, G. Y BEHAR, R. (2009) "Interpretaciones erradas del nivel de confianza en los intervalos de confianza y algunas explicaciones plausibles" en M. J. González; M. T. González y J. Murillo (Eds.), Investigación en Educación Matemática. Comunicaciones de los grupos de investigación. XIII Simposio de la SEIEM. Santander. 


\section{ANEXO 1. LA TAREA DE MODELACIÓN}

En la medicina, es común la utilización del saturómetro (Figura 1, parte izquierda), especialmente en centros asistenciales para monitorizar y realizar diagnósticos, con base en la saturación de oxígeno en la sangre y la frecuencia cardiaca del paciente.

La saturación de oxígeno es la cantidad de oxígeno que la hemoglobina lleva por todo el cuerpo, debido a que la hemoglobina es una molécula encargada del transporte de oxígeno. Mientras que, la frecuencia cardiaca es la cantidad de latido por minuto, promediado cada 5 a 20 segundos.

El saturómetro entrega en su pantalla tres elementos: Saturación de oxígeno en la sangre, frecuencia cardiaca y la curva pletismográfica de la señal de oximetría.

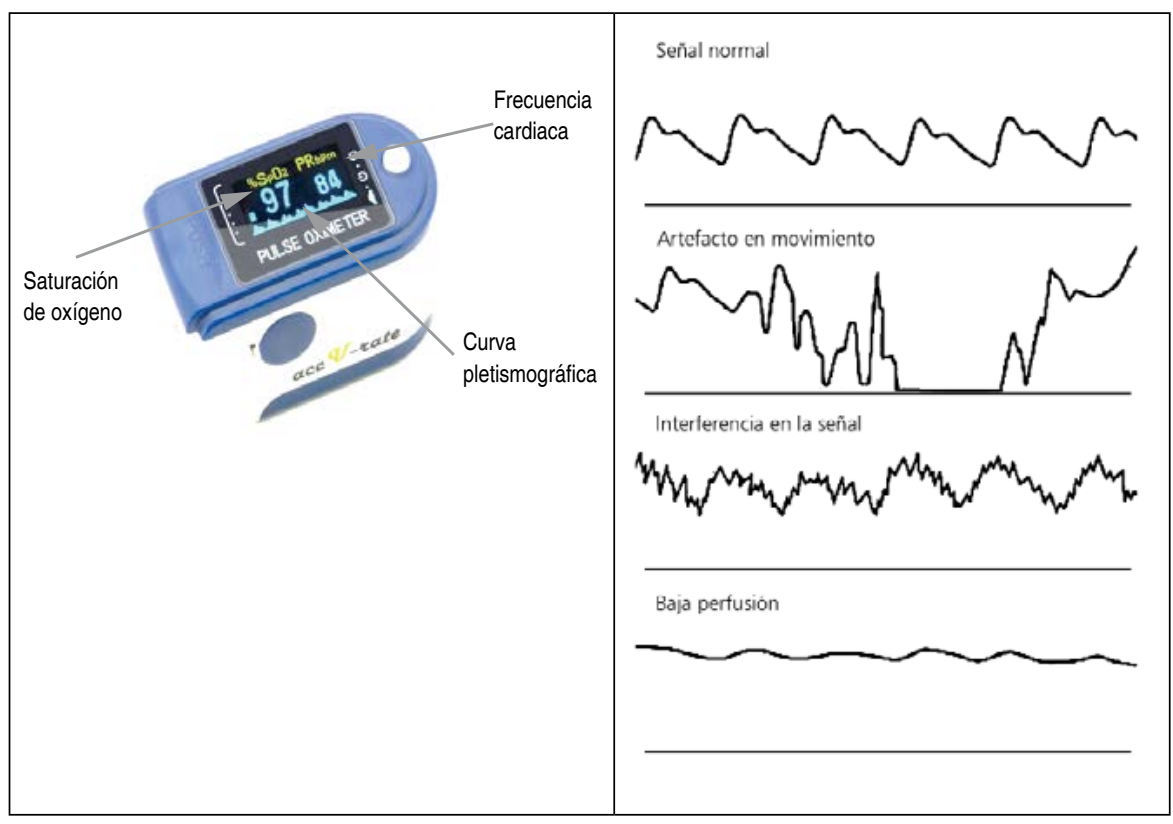

Fuente: Palacios, Álvarez, Schönffeldt, Céspedes, Gutiérrez y Oyarzún (2010). 
Para realizar una buena lectura de los datos que entrega el saturómetro, se debe tener cuidado de la curva pletismográfica. En la Figura 1 (parte derecha), se muestra cómo debe ser la curva al momento de la lectura (señal normal) y otras curvas que ocurren por diversos factores, que declaran que en ese momento no se debe hacer la lectura de datos. Cuando se toman los datos y se determina la certeza de ellos, estos se pueden interpretar según la Tabla 1.

Para el caso de la frecuencia, los niños recién nacidos hasta los dos años tienen una frecuencia cardiaca normal entre los 100 y 180; los infantes de dos a diez años, entre 60 y 140; en cambio, desde los diez años en adelante, el rango de normalidad es entre los 50 y los 100.

\section{Tabla 1}

Interpretación clínica de la saturación de oxígeno.

\begin{tabular}{cl}
\hline \% Saturación & \multicolumn{1}{c}{ Actuación } \\
\hline$>95 \%$ & No actuación inmediata. \\
\hline $95-90 \%$ & $\begin{array}{l}\text { Tratamiento inmediato y monitorización de la respuesta al mismo. } \\
\text { Según esta, valorar derivación al hospital. Los pacientes con } \\
\text { enfermedades respiratoria crónica toleran bien saturaciones en } \\
\text { torno a estos valores. }\end{array}$ \\
\hline$<90 \%$ & $\begin{array}{l}\text { Enfermo grave. Hipoxia severa. Oxigenoterapia + Tratamiento y } \\
\text { traslado al Hospital. }\end{array}$ \\
\hline$<80 \%$ & Valorar intubación y ventilación mecánica. \\
\hline
\end{tabular}

Fuente: Casado y Seco (2010).

\section{Tu misión}

Se deben formar grupos de tres estudiantes y establecer los siguientes roles: persona de estudio, operador del saturómetro, asistente (quien registra los datos entregados por el saturómetro) y observadores. 


\section{La organización}

1. La persona de estudio debe estar sentada con la mano apoyada en la mesa.

2. El operador del saturómetro debe colocar el saturómetro en el dedo índice de la persona de estudio.

3. El operador debe estar pendiente de la curva pletismográfica, para realizar una buena lectura de los datos.

4. El operario debe dictar al asistente los datos entregados por el saturómetro.

5. El operador debe colocar el saturómetro, hacer la lectura y sacar el saturómetro, repitiendo el procedimiento por 50 veces.

6. Los datos deben ser registrados en una planilla de Excel.

\section{Tu tarea}

Construya la tabla de frecuencia para la saturación de oxígeno y representa con un gráfico de barra.

a. ¿Cuál es la saturación de oxígeno referencial del compañero? (Explique su decisión).

b. ¿Cuál es la media de los datos?

c. Si consideramos la representación gráfica, la saturación de oxígeno referencial y la media de los datos. Entonces, ¿Cuál sería la saturación de oxígeno del compañero? y ¿cuál es su interpretación basándose en la Tabla 1?

d. A continuación, se entregan los datos de Javiera, compañera del cuarto medio A. ¿Cuál es la saturación de oxígeno de Javiera? y ¿Cuáles son las diferencias y similitudes, en base a la gráfica, saturación de oxígeno referencial y la media de los datos de Javiera, en comparación con los de su primer compañero? 


\begin{tabular}{|c|c|c|c|c|}
\hline \multirow[t]{2}{*}{$\mathrm{n}$} & \multicolumn{2}{|c|}{ Datos Javiera } & \multicolumn{2}{|c|}{ Datos a recopilar } \\
\hline & $\begin{array}{c}\text { Saturación } \\
\text { de oxígeno } \\
\text { (\%SpO2) }\end{array}$ & $\begin{array}{c}\text { Frecuencia } \\
\text { cardiaca } \\
\text { (PRbpm) }\end{array}$ & $\begin{array}{c}\text { Saturación } \\
\text { de oxígeno } \\
\text { (\%SpO2) }\end{array}$ & $\begin{array}{c}\text { Frecuencia } \\
\text { cardiaca } \\
\text { (PRbpm) }\end{array}$ \\
\hline 1 & 99 & 67 & & \\
\hline 2 & 95 & 76 & & \\
\hline 3 & 97 & 75 & & \\
\hline 4 & 96 & 66 & & \\
\hline 5 & 96 & 68 & & \\
\hline 6 & 96 & 75 & & \\
\hline 7 & 96 & 68 & & \\
\hline 8 & 98 & 64 & & \\
\hline 9 & 96 & 68 & & \\
\hline 10 & 98 & 71 & & \\
\hline 11 & 93 & 67 & & \\
\hline 12 & 97 & 70 & & \\
\hline 13 & 97 & 71 & & \\
\hline 14 & 96 & 67 & & \\
\hline 15 & 97 & 64 & & \\
\hline 16 & 98 & 68 & & \\
\hline 17 & 97 & 66 & & \\
\hline 18 & 97 & 68 & & \\
\hline 19 & 98 & 68 & & \\
\hline 20 & 97 & 65 & & \\
\hline 21 & 95 & 68 & & \\
\hline 22 & 98 & 64 & & \\
\hline 23 & 98 & 66 & & \\
\hline 24 & 98 & 74 & & \\
\hline 25 & 95 & 70 & & \\
\hline 26 & 96 & 68 & & \\
\hline
\end{tabular}




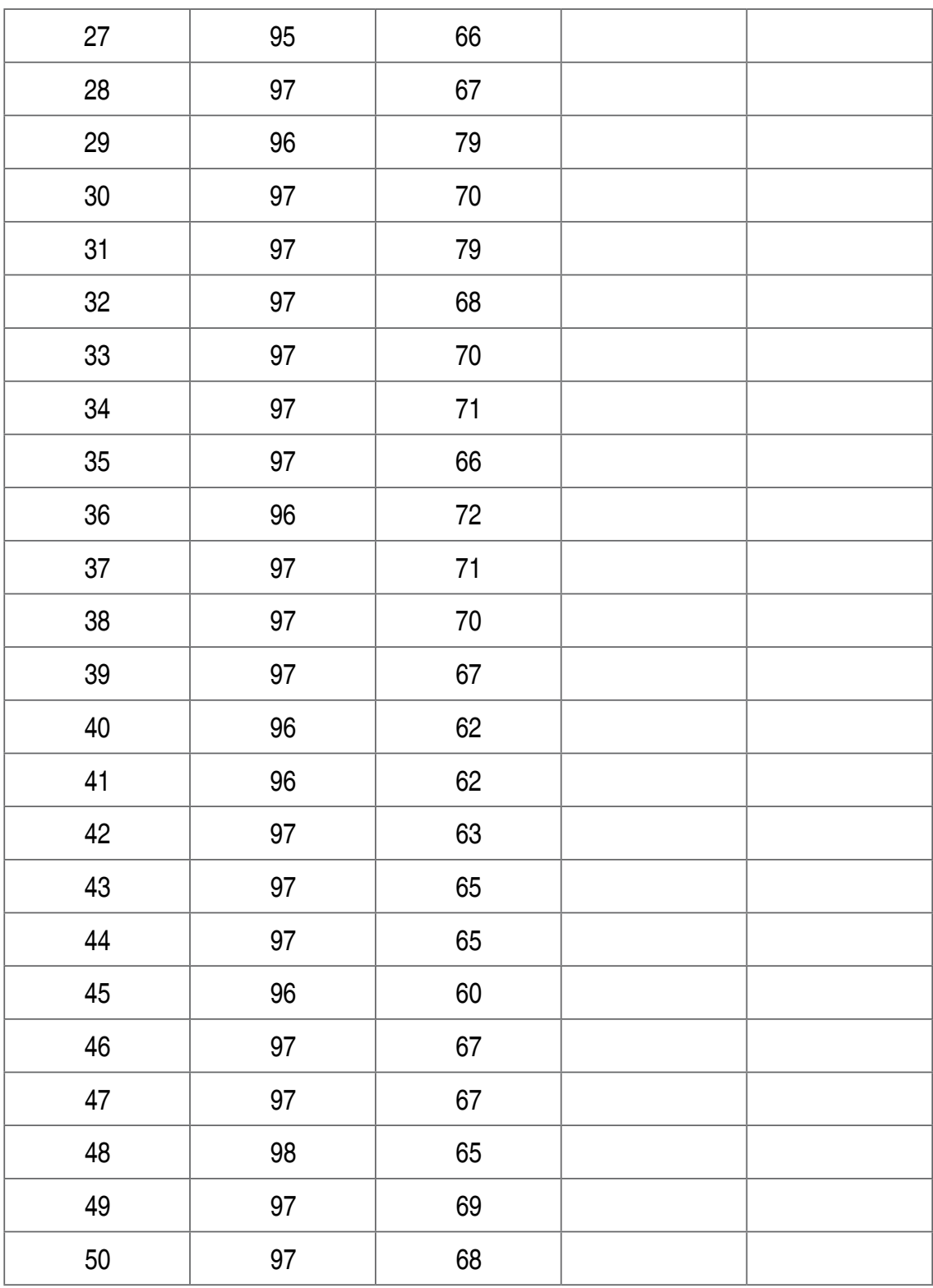

